\title{
Free Optimal Time Control Problem for a SEIR-Epidemic Model with Immigration of Infective
}

\author{
Mustapha Lhous \\ Laboratory of Modeling, Analysis, \\ Control and Statistics, Department of \\ Mathematics and Computer Science, \\ Faculty of Sciences Ain Chock, \\ Hassan II University of Casablanca, \\ B.P 5366 Maarif , \\ Casablanca, Morocco
}

\author{
Mostafa Rachik \\ Laboratory of Analysis Modelling \\ and Simulation, Department of \\ Mathematics and Computer Science, \\ Faculty of Sciences Ben M'sik, \\ Hassan II University of Casablanca, \\ B.P 7955 Sidi Othman, \\ Casablanca, Morocco
}

\author{
Abdelilah Larrache \\ Laboratory of Analysis Modelling \\ and Simulation, Department of \\ Mathematics and Computer Science, \\ Faculty of Sciences Ben M'sik, \\ Hassan II University of Casablanca, \\ B.P 7955 Sidi Othman, \\ Casablanca, Morocco
}

\begin{abstract}
In the present paper, we consider a mathematical model of a SEIR with immigration of infectives. The optimal control theory is applied to reduce the latent and infectious groups, increase the number of recovered individuals and this with an optimal cost. We use two controls representing the effort that reduces the contact between the infectious and susceptible individuals and a therapeutic treatment. We presents an approach that investigates a free terminal optimal time control witch give a minimum duration of a vaccination campaign. The Pontryagin's maximum principle is used to characterize the optimal controls and the optimal final time. We obtained an optimality system that we sought to solve numerically by an iterative discrete scheme that converges following an appropriate test similar the one related to the forward-backward sweep method.
\end{abstract}

\section{General Terms}

Vaccination, epidemiology

\section{Keywords}

SEIR-Epidemic model, Optimal control, Vaccination, Immigration

\section{INTRODUCTION}

The dynamics of the infectious diseases is an important research area in mathematical epidemiology. In epidemiology, it is well established that the immigration of people has an important role in the spread of an epidemic. Indeed, some diseases has been introduced into a population through the migration of infective individuals from outside into the host population like HIV, Ibola, SARS, Influenza, etc.

Epidemic models have been studied by many authors. Most of them are interested in the epidemic disease studied when spreading into a population with immigrants see [2], [7]-[9], [16], [17], [20] and [21].

Optimal control theory provides a valuable tool to begin to assess the trade-offs between vaccination and treatment strategies [7], [10], [12] and [13]. Optimal control is a mathematical technique de- rived from the calculus of variations

There are a number of different methods for calculating the optimal control for a specific mathematical model. For example, Pontryagin's maximum principe [18] allows the calculation of the optimal control for an ordinary equation model system with a given constraint.

In this paper, we consider the SEIR (Susceptible-Exposed-InfectedRemoved) epidemic model. We use an optimal control strategies to control the spread of infectious diseases by setting two control. The first control is introduced to reduces the contact between the infectious and susceptible individuals and the second control is considered as a therapeutic treatment. The two control are considered in order to minimize the number of exposed and infected individuals, increase the number of recovered individuals and this with a optimal cost.

However, the diseases immunization strategies are based on the conventional concept of time constant, while in practice, it is always advantageous to treat a disease as quickly as possible to minimize the negative effects of the disease on the patient's body. in addition, it's both difficult and expensive to implement vaccination for large population coverage in large time, especially while considering financial and logistical constraints. That's why we are interested to research for an optimal final time which allows us to attempt the aim of those strategies with an optimal cost. In this context, we set a free terminal time optimal control problem in the case of an SEIR epidemic model with vaccination.

The paper is organized as follows: In section 2, the SEIR epidemic model is described. The analysis of the optimal control strategies is presented, we formulate an optimal control problem with free terminal time to derive the optimal duration of vaccination in section 3. In section 4, we give the numerical method and the simulation results. Finally a conclusion is summarized in section 5 . 


\section{SEIR EPIDEMIC MODEL}

Consider the SEIR-type epidemic model

$$
\begin{aligned}
\dot{S}(t) & =-\mu S(t)+\omega R(t)-\beta \frac{S(t) I(t)}{N}+\mu N \\
\dot{E}(t) & =\beta \frac{S(t) I(t)}{N}-(\mu+\sigma) E(t) \\
\dot{I}(t) & =-(\mu+\gamma) I(t)+\sigma E(t) \\
\dot{R}(t) & =-(\mu+\omega) R(t)+\gamma I(t)
\end{aligned}
$$

subject to initial conditions $S(0) \geq 0, E(0) \geq 0, I(0) \geq 0$ and $R(0) \geq 0$.

In such a SEIR-model, $\mathrm{N}$ is the total population, $\mu$ is the rate of deaths from causes unrelated to the infection, $\omega$ is the rate of losing immunity, $\beta$ is the transmission constant (with the total number of infections per unity of time at time t being $\left.\beta \frac{S(t) I(t)}{N}\right), \sigma^{-1}$ and $\gamma^{-1}$ are, respectively, the average duration of latent and infective periods, see [3] and [5]. All the above parameters are assumed to be nonnegative.

Schematically, the flow between compartments is represented as

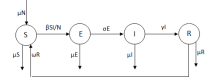

Assertion 1: The SEIR model (1)-(4) fulfils the constant population through time constraint, i.e.:

$$
N(t)=S(t)+E(t)+I(t)+R(t)=N(0)=N>0 .
$$

Into the model (1)-(4) we include two controls $u$ and $v$ that represent, respectively, the effort that reduces the contact between the infectious and susceptible individuals, and the rate at which infectious individuals are treated at each time period. We assume that $v I$ individuals per time are removed the infected class and added to the recovred class. The mathematical system with controls is given by nonlinear differential equations

$$
\begin{aligned}
\dot{S}(t) & =-\mu S(t)+\omega R(t)-(1-u) \beta \frac{S(t) I(t)}{N}+\mu N \\
\dot{E}(t) & =(1-u) \beta \frac{S(t) I(t)}{N}-(\mu+\sigma) E(t) \\
\dot{I}(t) & =-(\mu+\gamma+v) I(t)+\sigma E(t) \\
\dot{R}(t) & =-(\mu+\omega) R(t)+(\gamma+v) I(t)
\end{aligned}
$$

with $S(0) \geq 0, E(0) \geq 0, I(0) \geq 0$ and $R(0) \geq 0$, are given.

\section{THE OPTIMAL VACCINATION}

Optimal control techniques are of great use in developing optimal strategies to control various kinds of diseases. Our goal is to minimize the number of exposed and infected individuals and to increase the number of recovered individuals during the course of an epidemic and to minimize the cost of this strategy and this with a minimal duration of vaccination. To solve this problem we use optimal control theory.

Now, we consider an optimal control problem to minimize the objective functional

$J(u, v, T)=\int_{0}^{T}\left[E(t)+I(t)-R(t)+\frac{A_{1}}{2} u^{2}(t)+\frac{A_{2}}{2} v^{2}(t)\right] d t+\phi(T)$

subject to system (6)-(9), where the parameters $A_{1}$ and $A_{2}$ are positive weights parameters which is associated with the control $u(t)$ and $v(t)$ respectively. T represent the duration of the vaccination. $\phi$ is a positive increasing function such that $\lim \phi(t)=+\infty$.

In other words, we seek the optimal control $\left(u^{*}, v^{*}\right)$ and an optimal terminal time $T^{*}$ such that

$$
J\left(u^{*}, v^{*}, T^{*}\right)=\min \left\{J(u, v, T) \mid(u, v) \in \mathcal{U}_{a d}, T \in \mathbb{R}^{+}\right\}
$$

Where $\mathcal{U}_{a d}$ is the set of admissible controls defined by

$$
\begin{aligned}
\mathcal{U}_{a d}= & \{(u, v) \mid u \text { and } v \text { are Lebesgue measurable, } \\
& 0 \leq u(t) \leq 1,0 \leq v(t) \leq 1, t \in[0, T]\} .
\end{aligned}
$$

In order to find an optimal solution, first we find the Lagrangian and Hamiltonian for the optimal control problem 10 . In fact, the Lagrangian of the optimal problem is given by

$$
L(E, I, u, v)=E(t)+I(t)-R(t)+\frac{A_{1}}{2} u^{2}(t)+\frac{A_{2}}{2} v^{2}(t) .
$$

We seek the minimal value of the Lagrangian. To accomplish this, we define the Hamiltonian $H$ for the control problem:

$H=L(E, I, u, v)+\lambda_{1} \frac{d S(t)}{d t}+\lambda_{2} \frac{d E(t)}{d t}+\lambda_{3} \frac{d I(t)}{d t}+\lambda_{4} \frac{d R(t)}{d t}$

where $\lambda_{1}, \lambda_{2}, \lambda_{3}$ and $\lambda_{4}$ are the adjoint functions to be determined suitably.

\subsection{Characterization of the optimal control}

In the previous section we show the existence of an optimal control which minimize the functional (10) subject to system (6)-(9). In order to derive the necessary conditions for this optimal control, we apply Pontryagin's maximum principle to the Hamiltonian H.

Theorem 1. Let $S^{*}(t), E^{*}(t), I^{*}(t)$ and $R^{*}(t)$ be optimal state solutions with associated optimal control pair $\left(u^{*}(t), v^{*}(t)\right)$ for the optimal control problem (11). Then, there exist adjoint variables $\lambda_{1}, \lambda_{2}, \lambda_{3}$ and $\lambda_{4}$, that satisfy

$$
\left\{\begin{array}{l}
\dot{\lambda}_{1}=\mu \lambda_{1}+\beta \frac{I^{*}}{N}(1-u)\left(\lambda_{1}-\lambda_{2}\right) \\
\dot{\lambda}_{2}=-1+\mu \lambda_{2}+\sigma\left(\lambda_{2}-\lambda_{3}\right) \\
\dot{\lambda}_{3}=-1+\beta \frac{S^{*}}{N}(1-u)\left(\lambda_{1}-\lambda_{2}\right)+\mu \lambda_{3}+(\gamma+v)\left(\lambda_{3}-\lambda_{4}\right) \\
\dot{\lambda}_{4}=1+\mu \lambda_{4}+\omega\left(\lambda_{4}-\lambda_{1}\right)
\end{array}\right.
$$

with transversality conditions

$$
\lambda_{i}(T)=0, \quad i=1,2,3,4 .
$$

Furthermore, the optimal control $\left(u^{*}, v^{*}\right)$ is given by

$$
\begin{gathered}
u^{*}=\max \left\{\min \left\{\frac{\beta S I\left(\lambda_{2}-\lambda_{1}\right)}{N A_{1}}, 1\right\}, 0\right\} \\
v^{*}=\max \left\{\min \left\{\frac{I\left(\lambda_{3}-\lambda_{4}\right)}{A_{2}}, 1\right\}, 0\right\}
\end{gathered}
$$

and the optimal final time is given by

$\frac{\partial \phi}{\partial t}\left(T^{*}\right)=-E\left(T^{*}\right)-I\left(T^{*}\right)+R\left(T^{*}\right)-\frac{A_{1}}{2} u^{2}\left(T^{*}\right)-\frac{A_{2}}{2} v^{2}\left(T^{*}\right)$.

PROOF. The existence of optimal control can be proved by using the result from [19] (see theorem 2.1). The adjoint equation and transversality conditions can be obtained by using Pontryagin's 
Maximum Principle such that

$$
\left\{\begin{array}{l}
\dot{\lambda}_{1}=-\frac{\partial H}{\partial S}, \lambda_{1}(T)=0 \\
\dot{\lambda}_{2}=-\frac{\partial H}{\partial E}, \lambda_{2}(T)=0 \\
\dot{\lambda}_{3}=-\frac{\partial H}{\partial I}, \lambda_{3}(T)=0 \\
\dot{\lambda}_{4}=-\frac{\partial H}{\partial R}, \lambda_{4}(T)=0
\end{array}\right.
$$

The optimal control pair $\left(u^{*}, v^{*}\right)$ can be solve from the optimality condition

$$
\frac{\partial H}{\partial u}=0 \text { and } \frac{\partial H}{\partial v}=0 .
$$

By the bounds in $U$ of the control, it is easy to obtain $\left(u^{*}, v^{*}\right)$ in the form of (15)- 16$)$. The transversality condition for $\mathrm{T}$ to be the optimal terminal time can be stated as

$$
H\left(T^{*}, E^{*}, I^{*}, R^{*}, u^{*}, v^{*}\right)+\frac{\partial \phi}{\partial t}\left(T^{*}\right)=0
$$

Thus, $T^{*}$ may be rewritten as in 17 .

The optimal control and the state are found by solving the optimality system, which consists of the state system (6)-(9) with initial conditions at $t=0$, the adjoint system (13) with the final conditions (14) and the characterization of the optimal control [15, 16. So the optimality system is given by

$$
\left\{\begin{aligned}
\dot{S}^{*}(t)= & -\mu S^{*}(t)+\omega R^{*}(t)-(1- \\
& \left.\max \left\{\min \left\{\frac{\left(\lambda_{2}-\lambda_{1}\right) \beta S^{*}(t) I^{*}(t)}{N A_{1}}, 1\right\}, 0\right\}\right) \beta \frac{S^{*}(t) I^{*}(t)}{N}+\mu N \\
\dot{E}^{*}(t)= & \left(1-\max \left\{\min \left\{\frac{\left(\lambda_{2}-\lambda_{1}\right) \beta S^{*}(t) I^{*}(t)}{N A_{1}}, 1\right\}, 0\right\}\right) \beta \frac{S^{*}(t) I^{*}(t)}{N} \\
& -(\mu+\sigma) E^{*}(t) \\
\dot{I}^{*}(t)= & -\left(\mu+\gamma+\max \left\{\min \left\{\frac{\left(\lambda_{3}-\lambda_{4}\right) I^{*}(t)}{A_{2}}, 1\right\}, 0\right\}\right) I^{*}(t) \\
& +\sigma E^{*}(t) \\
\dot{R}^{*}(t)= & -(\mu+\omega) R^{*}(t)+(\gamma+ \\
& \left.\max \left\{\min \left\{\frac{\left(\lambda_{3}-\lambda_{4}\right) I^{*}(t)}{A_{2}}, 1\right\}, 0\right\}\right) I^{*}(t) \\
\dot{\lambda}_{1}= & \mu \lambda_{1}+\beta \frac{I^{*}}{N}\left(1-\max \left\{\min \left\{\frac{\left(\lambda_{2}-\lambda_{1}\right) \beta S^{*}(t) I^{*}(t)}{N A_{1}}, 1\right\}, 0\right\}\right) \\
& \left(\lambda_{1}-\lambda_{2}\right) \\
\dot{\lambda}_{2}= & -1+\mu \lambda_{2}+\sigma\left(\lambda_{2}-\lambda_{3}\right) \\
\dot{\lambda}_{3}= & -1+\beta \frac{S^{*}}{N}\left(1-\max \left\{\min \left\{\frac{\left(\lambda_{2}-\lambda_{1}\right) \beta S^{*}(t) I^{*}(t)}{N A_{1}}, 1\right\}, 0\right\}\right) \\
& \left(\lambda_{1}-\lambda_{2}\right)+\mu \lambda_{3}+(\gamma+ \\
& \left.\max \left\{\min \left\{\frac{\left(\lambda_{3}-\lambda_{4}\right) I^{*}(t)}{A_{2}}, 1\right\}, 0\right\}\right)\left(\lambda_{3}-\lambda_{4}\right) \\
\dot{\lambda}_{4}= & 1+\mu \lambda_{4}+\omega\left(\lambda_{4}-\lambda_{1}\right)
\end{aligned}\right.
$$

with $\lambda_{1}(T)=0, \lambda_{2}(T)=0, \lambda_{3}(T)=0, \lambda_{4}(T)=0, S(0)=S_{0}$, $E(0)=E_{0}, I(0)=I_{0}$ and $R(0)=R_{0}$.

\section{NUMERICAL SIMULATION}

\subsection{The improved GSSI1 methode}

The resolution of the optimality system $(19)$ is created improving the Gauss Seidel - like implicit finite-difference method developed in ([10]) and denoted GSSI1 method. It consist on descretizing the interval $[0,1]$ by the point $t_{k}=k l(k=0,1 \ldots, n)$, where $l$ is the time step. Next, we define the state and adjoint variables $S(t), E(t), I(t), R(t), \lambda_{1}(t), \lambda_{2}(t), \lambda_{3}(t), \lambda_{4}(t)$ and the control $u(t), v(t)$ in terms of nodal points $S^{k}, E^{k}, I^{k}, R^{k}, \lambda_{1}^{k}, \lambda_{2}^{k}, \lambda_{3}^{k}, \lambda_{4}^{k}$, $u^{k}$ and $v^{k}$ with $S^{0}, E^{0}, I^{0}, R^{0}, \lambda_{1}^{0}, \lambda_{2}^{0}, \lambda_{3}^{0}, \lambda_{4}^{0}, u^{0}$ and $v^{0}$ as the state and adjoint variables and the control at initial time $t=0 . S^{n}$, $E^{n}, I^{n}, R^{n}, \lambda_{1}^{n}, \lambda_{2}^{n}, \lambda_{3}^{n}, \lambda_{4}^{n}, u^{n}$ and $v^{n}$ as the state and adjoint variables and the control at final time $t=T$.

As it well known, the approximation of the time derivative by its first-order forward-difference is given, for the first state variable
$S(t)$ by $\frac{d S(t)}{d t}=\lim _{l \rightarrow 0} \frac{S(t+l)-S(t)}{l}$. Then, we use the following scheme

$$
\begin{aligned}
\frac{S^{k+1}-S^{k}}{l} & =-\mu S^{k}+\omega R^{k}-\left(1-u^{k}\right) \beta \frac{S^{k} I^{k}}{N}+\mu N \\
\frac{E^{k+1}-E^{k}}{l} & =\left(1-u^{k}\right) \beta \frac{S^{k} I^{k}}{N}-(\mu+\sigma) E^{k} \\
\frac{I^{k+1}-I^{k}}{l} & =-\left(\mu+\gamma+v^{k}\right) I^{k}+\sigma E^{k} \\
\frac{R^{k+1}-R^{k}}{l} & =-(\mu+\omega) R^{k}+\left(\gamma+v^{k}\right) I^{k}
\end{aligned}
$$

And we approximate the time derivative of the adjoint variables and we use the appropriated scheme as follows

$$
\begin{aligned}
\frac{\lambda_{1}^{k+1}-\lambda_{1}^{k}}{l}= & \mu \lambda_{1}^{k}+\beta \frac{I^{* k}}{N}\left(1-u^{k}\right)\left(\lambda_{1}^{k}-\lambda_{2}^{k}\right) \\
\frac{\lambda_{2}^{k+1}-\lambda_{2}^{k}}{l}= & -1+\mu \lambda_{2}^{k}+\sigma\left(\lambda_{2}^{k}-\lambda_{3}^{k}\right) \\
\frac{\lambda_{3}^{k+1}-\lambda_{3}^{k}}{l}= & -1+\beta \frac{S^{* k}}{N}\left(1-u^{k}\right)\left(\lambda_{1}^{k}-\lambda_{2}^{k}\right)+ \\
& \mu \lambda_{3}^{k}+\left(\gamma+v^{k}\right)\left(\lambda_{3}^{k}-\lambda_{4}^{k}\right) \\
\frac{\lambda_{4}^{k+1}-\lambda_{4}^{k}}{l}= & 1+\mu \lambda_{4}^{k}+\omega\left(\lambda_{4}^{k}-\lambda_{1}^{k}\right)
\end{aligned}
$$

\subsection{Numerical result}

In this section we present the results obtained by solving numerically the optimality system given by theorem 1 . This system consists of the state system, adjoint system, initial and final time conditions, and the control characterization.

The optimality systems is solved based on an iterative discrete scheme that converges following an appropriate test similar the one related to the Forward-Backward Sweep Method (FBSM). The state system with an initial guess is solved forward in time and then the adjoint system is solved backward in time because of the transversality conditions. Afterwards, we update the optimal control values using the values of state and adjoint variables obtained at the previous steps. Finally, we execute the previous steps till a tolerance criterion is reached.

We consider an example of an epidemic described by the SEIR model (6)-(9) with parameter values: $\mu=0.16 \times 10^{-5}$ per day (p.d.), $\beta=0.098$ p.d., $\omega=10^{-4}$ p.d., $\sigma=0.182$ p.d., $\gamma=2.7 \times 10^{-4}$ p.d. and a total population of $N=7000$. The initial condition for the individual population are given by: $S(0)=4000, E(0)=2000, I(0)=1000, R(0)=0$. We use $A_{1}=250, A_{2}=1.5 \times 10^{5}$ and $\phi(t)=2 \times 10^{-4} t^{5}$.

Considering the critical level of vaccination, we give a optimal control sufficient to reduce the number of infected and exposed individuals and to increase the removed individuals.

We give a final time sufficient to eradicate disease. By this way, numerical simulations suggest 40 days as final time of the vaccination campaign see Figure 1. 


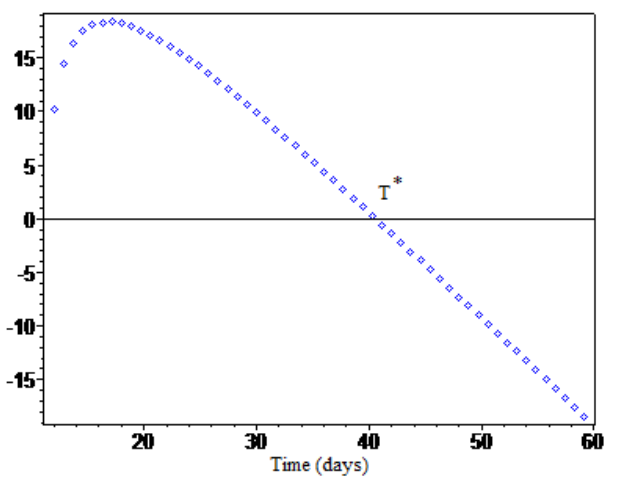

Fig.1. The optimal final time $T^{*}$.

The graphs below, allow us to compare changes in the number of infected, exposed and removed individuals before and after the introduction of control.

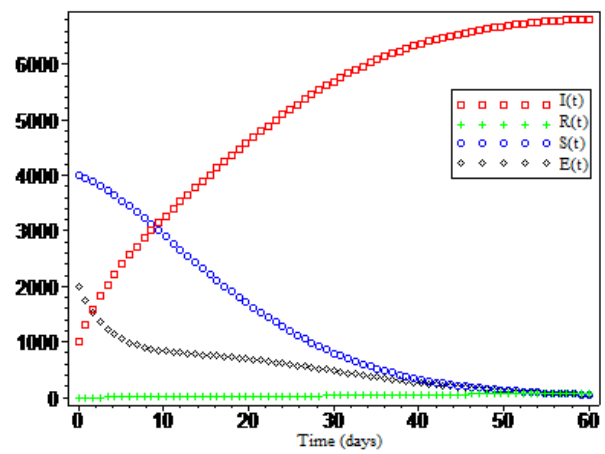

Fig.2. Time evolution of the individual populations without controls.

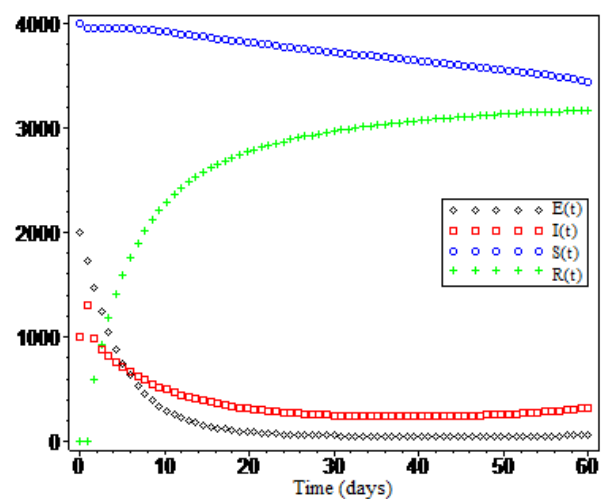

Fig.3. Time evolution of the individual populations with controls.

Figure 4 also shows the effect of control by indicating that the number of exposed individuals decreases more rapidly during the vaccination campaign.

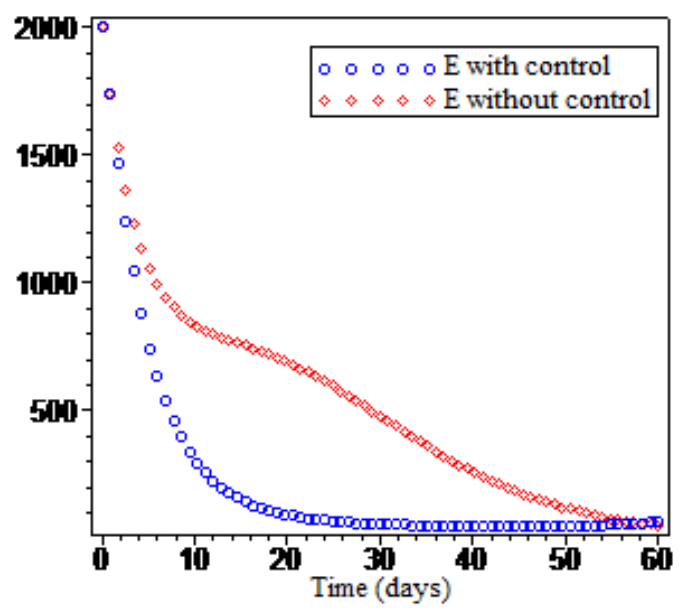

Fig.4. The Exposed individuals with and without controls

Figure 5 gives an example of the evolution of the number of infected individuals with and without control. We notice that in absence of control, the infected group grew to extremely high levels and in presence of the control, this group decrease greatly.

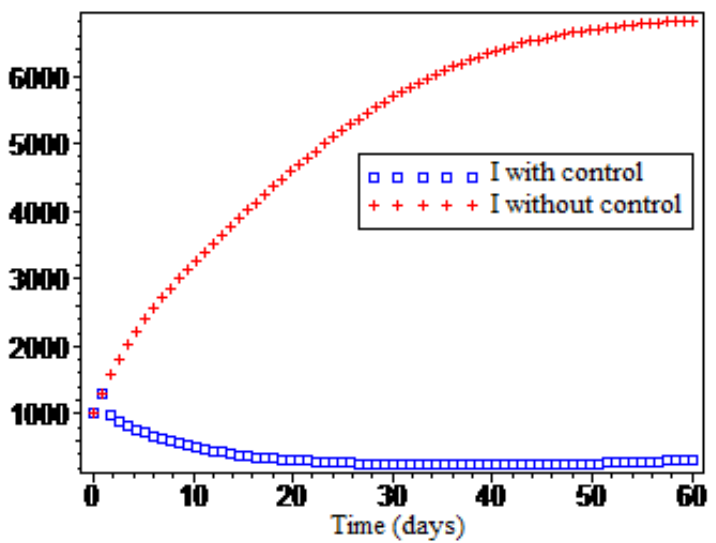

Fig.5. The Infectious individuals with and without controls.

Figure 6, show that the number of people removed with control begins to grow more than without control. In the end of the vaccination campaign, the number of recovered individuals population grew to extremely high levels.

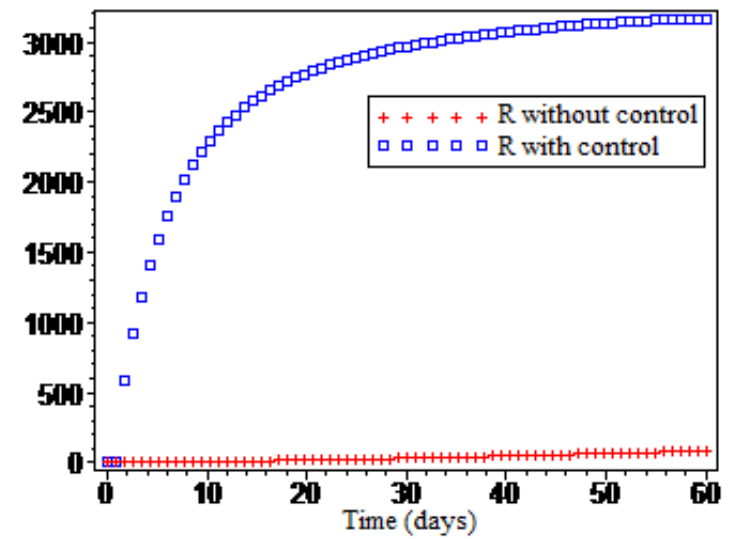


Fig.6. The Recovered individuals with and without controls.

Finally, Figure 7 and Figure 8 gives the optimal control $u^{*}$ and $v^{*}$ sufficient to eradicate disease.

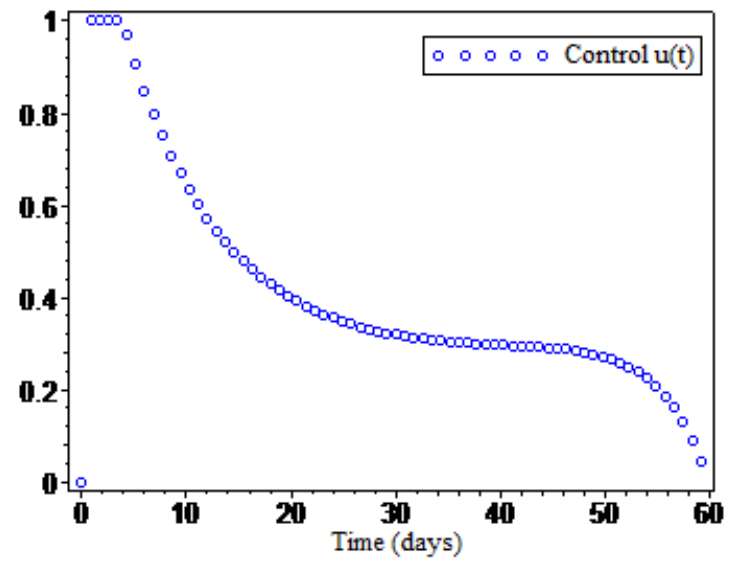

Fig.7. The optimal control $u^{*}(t)$.

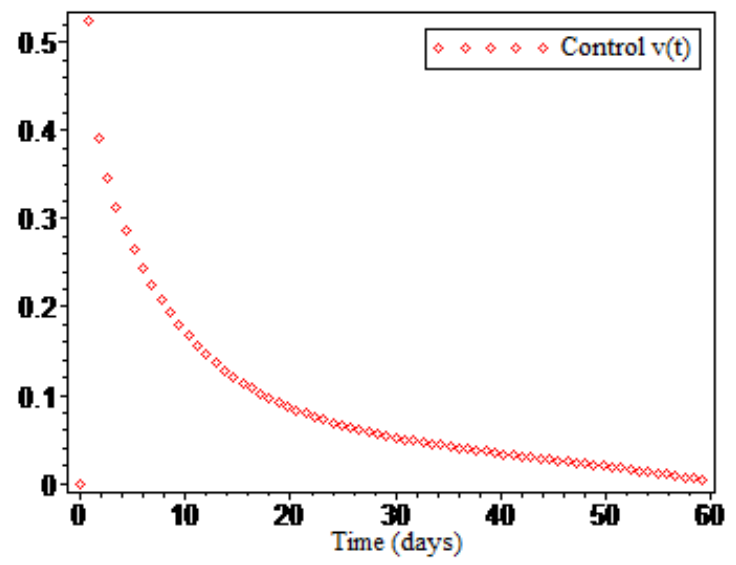

Fig.8. The optimal control $v^{*}(t)$

\section{CONCLUSION}

In this paper, we consider a mathematical model of a SEIR with immigration of infectives. The optimal control has been considered and two controls have been introduced representing the effort that reduces the contact between the infectious and susceptible individuals and a therapeutic treatment. A free terminal optimal time control is also investigated. The Pontryagin's maximum principle is used to characterize the optimal controls and the optimal final time. A comparison between individuals with optimal control and no control is presented. A numerical simulation has been given to demonstrate the use of the obtained results.

\section{REFERENCES}

[1] Birkhoff, G, Rota, G, C. 1989. Ordinary Differential Equations, 4th ed. John Wiley and Sons, New York.

[2] Brauer, F, Van den Driessche, P. 2001. Models for transmission of disease with immigration of infectives, Mathematical Biosciences, 171, 154-143.
[3] De la Sen, M, Alonso-Quesada, S. 2010. On vaccination control tools for a general SEIR-epidemic model, 18th Mediterranean Conference on Control and Automation (MED'10), pp. 1322-1328.

[4] De la Sen, M, Alonso-Quesada, S. June 2010. A simple vaccination controlstrategy for the SEIR epidemic model, in Proceeding of the 5th IEEE International Conference on Management of Innovation and Technology, pp. 1037-1044.

[5] De la Sen, M, Ibeas, A, Alonso-Quesada, S. 2011. Feedback linearization-based vaccination control strategies for true-mass action type SEIR epidemic models, Nonlinear analysis: Modelling and Control, Vol. 16, No. 3, 283-314.

[6] De la Sen, M, Ibeas, A, Alonso-Quesada, S. 2012. On vaccination controls for the SEIR epidemic model, Commun Nonlinear Sci Numer Simulat, Vol. 17, 2637-2658.

[7] El hia, M, Balatif, O, Rachik, M, Bouyaghroumni, J. 2013. Application of optimal control theory to an SEIR model with immigration of infectives, International Journal of Computer Science, Vol. 10, Issue 2. No. 2, 230-236.

[8] Jia, Z, W, Tang, G, Y, Jin, Z et al. 2008. Modeling the impact of immigration on the epidemiology of tuberculosis, Theoretical Population Biology, vol. 73, no. 3, 437-448.

[9] Zhang, J, Li, J, Ma, Z. 2006. Global dynamics of an SEIR epidemic model with immigration of different compartments, Acta Mathematica Scientia, 26B(3), 551-567.

[10] Gumel, A, B, Shivakumar, P, N, Sahai, B, M. 2001. A mathematical model for the dynamics of HIV-1 during the typical course of infection, Nonlinear Analysis. 47, 1773-1783.

[11] Kalivianakis, M, Mous, S, L, Grasman. 1994. Reconstruction of the seasonally varying contact rate for measles, Mathematical Biosciences, Vol. 124, No. 2, 225-234.

[12] Laarabi, H, Labriji, E, Rachik, M, Kaddar, A. 2012. Optimal control of an epidemic model with a saturated incidence rate, Nonlinear Analysis: Modelling and Control, Vol. 17, No. 4, 448-459.

[13] Laarabi, H, Rachik, M, El Kahlaoui, O, Labriji, E. 2013. Optimal Vaccination Strategies of an SIR Epidemic Model with a Saturated Treatment, Universal Journal of Applied Mathematics, 1(3), 185-191.

[14] Lukes, D, L: Differential Equations. 1982. Classical to Controlled, Math. Sci. Eng. 162, Academic Press, New York

[15] Mukhopadhyay, B, Bhattacharyya, R. 2007. Existence of epidemic waves in a disease transmission model with two-habitat population, Int. J. Syst. Sci., Vol. 38, No. 9, 699-707.

[16] Naresh, R, Tripathi, A, Sharma, D. 2009. Modelling and analysis of the spread of AIDS epidemic with immigration of HIV infectives, Mathematical and Computer Modelling, 49(5-6), 880-892.

[17] Piccolo, C, Billings, L. 2005. The Effect of Vaccinations in an Immigrant Model, Mathematical and Computer Modelling 42, 299-291.

[18] Pontryagin, L, S, Boltyanskii, V, G, Gamkrelidze, R, V, Mishchenko, E, F. 1962. The Mathematical Theory of Optimal Processes, Wiley, New York.

[19] Raj joshi, H, Lenhart, S, Li, M, Y, Wang, L. 2006. Optimal control methods applied to disease models-Contemporary Mathematics, Volume 410.

[20] Wang, W, Xin, J, Zhang, F. 2010. Persistence of an SEIR Model with Immigration Dependent on the Prevalence of Infection, Discrete Dynamics in Nature and Society, Article ID 727168, 7 pages.

[21] Zhou, Y, Khan, K, Feng, Z, Wu, J. 2008. Projection of tuberculosis incidence with increasing immigration trends, Journal of Theoretical Biology, vol. 254, no. 2, 215-228. 